\title{
Distributed Sensing and Estimation Under Communication Constraints
}

\author{
Yasamin Mostofi ${ }^{\dagger}$ and Richard M. Murray ${ }^{\dagger \dagger}$ \\ ${ }^{\dagger}$ University of New Mexico ${ }^{\dagger \dagger}$ California Institute of Technology
}

\begin{abstract}
In this paper we consider the impact of imperfect communication links on distributed sensing and estimation in mobile networks. First we will find optimum sensing regions and sensor positions under communication constraints. We will show that the optimum sensor configuration consists of overlapping sensing regions. We will then show how the nodes can achieve the optimum configuration in a distributed manner.
\end{abstract}

\section{INTRODUCTION}

The advent of cheap embedded units that are equipped with sensing, communication, processing and actuation capabilities has recently created considerable interest in networked sensing and control. Such networks have a wide range of applications such as environmental monitoring, surveillance and security, smart homes and factories, target tracking and military systems.

In this paper we are considering a scenario where a group of mobile units are given the task of cooperatively estimating an event of interest in a region. Each node has limited observation capabilities. Therefore, they can only achieve the task in a networked manner. We are interested in finding the optimum sensor positions and sensing regions when considering non-ideal communication links among the nodes. Optimum sensor configuration, without communication considerations, are easy to formulate, are closely related to locational optimization problems [1], [2] and are typically solved using Voronoi cells. Authors in [3] have provided a comprehensive treatment of centroidal Voronoi cells, indicating their applications in several different fields. Lloyd algorithm, a classic algorithm in quantization theory, provides an iterative decent way of achieving the optimum configuration under certain conditions [4], [5]. J. Cortes et al. have extended this algorithm to mobile sensor networks, without considering communication noise [6].

Wireless communications can play a key role in the overall performance of such systems as sensor measurements are exchanged over wireless links. In a distributed scenario, there is no central node responsible for collecting all the measurements. Therefore, the positions of the nodes not only affect their sensing but also impact the quality of communication among the nodes and therefore the overall networked sensing performance. Authors in [7], [8], [9], [10] have looked at the impact of communication channels on Kalman filtering over a wireless link and the conditions required for stability. Authors in [11], [12], [13], [14], [15] have looked at the impact of different aspects of a communication link such as noise, quantization, fading, medium access and packet loss on wireless control of a mobile sensor. Authors in [16] proposed algorithms for coordination of mobile vehicles with limited-range interactions.

Impact of communication channels on the optimum sensor configuration in networked sensing and estimation, however, has not been studies extensively. We are also interested in achieving the optimum configuration in a distributed manner. By "distributed", we are referring to the scenarios where each sensor, independently, makes a local decision about where to go next, based on the information that it receives only from its neighbors. Authors in [17] have considered the impact of non-ideal communication links on decentralized motion-planning in target tracking networks. They showed, through simulations, that the optimum configuration can change considerably when considering non-perfect links. A mathematical foundation that indicates optimum sensing regions and sensor positions, however, is still lacking. It is the goal of this paper to find the impact of communication links on optimum sensing configuration in distributed networks analytically. We will show that the new optimum configuration consists of overlapping sensing regions. Based on the analysis, we furthermore propose a distributed algorithm to achieve the optimum configuration.

\section{System Model}

Consider $n$ mobile sensors with the task of cooperatively sensing and estimating an event that will occur in region $Z$. $Z$ represents the region of interest in $\mathbb{R}^{N}$. Let $p: Z \rightarrow \mathbb{R}_{+}$ represent the probability density function that corresponds to the probability of the occurrence of the event over region $Z$. We are interested in finding the optimum "sensing regions" and "sensor positions", as well as distributed ways of achieving the optimum configuration from any initial positions.

Let $\Pi_{i} \subset Z$ represent sensing region of the $i^{\text {th }}$ sensor. This means that if an event occurs in $\Pi_{i}$, the $i^{\text {th }}$ sensor relies on its own observation of the event whereas for an event that occurs outside $\Pi_{i}$, the $i^{\text {th }}$ sensor relies on receiving the corresponding measurement from other sensors. An example is shown in Fig. 1 for $\mathbb{R}^{2}$, where $x_{i}$ represents the position of the $i^{\text {th }}$ sensor.

Remark II.1: It is important that all the sensors have an estimate of the occurred event since they may want to take a specific action such as repositioning themselves. 
Therefore, quality of communication links can affect the overall performance considerably as sensors exchange their measurements over wireless links. This does not necessarily mean that each sensor needs to transmit to the rest of the network when an event occurs, as we shall see later in the paper.

Let $U \in \mathbb{R}$ represent the measurable feature of the event of interest. ${ }^{1}$ Consider the case that an event happened at location $x$ in $\Pi_{i}$. Then the following will represent the measurement of the $i^{\text {th }}$ sensor: $\hat{U}_{i}=U+w_{i}$ for $x \in \Pi_{i}$, where $w_{i}$ represents zero-mean observation noise of the $i^{\text {th }}$ sensor with variance of $\sigma_{w_{i}}^{2}$. The measurement quality of the $i^{\text {th }}$ sensor degrades as $\left\|x-x_{i}\right\|$ increases, where $\|$. represents the Euclidean distance. We have $\sigma_{w_{i}}^{2}=f(\| x-$ $x_{i} \|$ ), where $f($.$) represents a non-decreasing function. Let$ $\Pi_{i, j} \subset Z$ represent the region in which the $i^{\text {th }}$ sensor relies on receiving the estimate of $U$ from the $j^{\text {th }}$ sensor if $x \in \Pi_{i, j}$. Naturally $\Pi_{i, i}=\Pi_{i}$.

Remark II.2: For each $i, \Pi_{i, j}$ s for $1 \leq j \leq n$ partition $Z$, i.e. $\forall i \bigcup_{j=1}^{n} \Pi_{i, j}=Z$ and $\Pi_{i, j} \cap \Pi_{i, z}=\emptyset$ for $j \neq z$.

Remark II.3: Note that $\Pi_{i, j} \neq \Pi_{j}$ for $j \neq i$ under communication constraints. We will elaborate more on this in the subsequent sections.

Consider an event that occurred in $\Pi_{i, j}$, where $j \neq i$. Then the following will represent the estimate of the $i^{\text {th }}$ sensor after communicating with the $j^{\text {th }}$ one:

$$
\hat{U}_{i}=U+w_{j}+v_{i, j} \quad \text { if } x \in \Pi_{i, j},
$$

where $v_{i, j}$ represents zero-mean communication noise associated with the transmission of the measurement from the $j^{\text {th }}$ sensor to the $i^{\text {th }}$ one. The transmission from the $j^{\text {th }}$ sensor to the $i^{\text {th }}$ one may not necessarily be a direct one. Depending on the locations of the sensors, it may be more cost effective to have a multi-hop routing, as we shall explore later in the paper. Let $\sigma_{v_{i, j}}^{2}$ represent the communication noise variance associated with $v_{i, j}$. Let $\bar{D}_{i}$ represent the estimation error variance of the $i^{\text {th }}$ sensor, averaged over the probability distribution of the event. We will have,

$$
\begin{aligned}
\bar{D}_{i} & =\int_{x \in \Pi_{i}} f\left(\left\|x-x_{i}\right\|\right) p(x) d x \\
& +\sum_{j \neq i} \int_{x \in \Pi_{i, j}}\left(f\left(\left\|x-x_{j}\right\|\right)+\sigma_{v_{i, j}}^{2}\right) p(x) d x .
\end{aligned}
$$

The overall estimation error variance of the network will then be as follows: $\bar{D}=\sum_{i=1}^{n} \bar{D}_{i} / n$. Then the optimum sensing regions, $\Pi_{i}$ and $\Pi_{i, j}$, and sensor positions, $x_{i}$, are those that minimize $\bar{D}$.

\section{A. Observation and Communication Noises}

In this paper, we take $f(\|s\|)=\xi\|s\|^{2}$ for an arbitrary $s$. Let $\sigma_{v_{i, j, d i r e c t}}^{2}$ represent the variance of the communication

\footnotetext{
${ }^{1}$ The analysis can be easily extended to $U \in \mathbb{R}^{M}$ for $M \neq 1$.
}

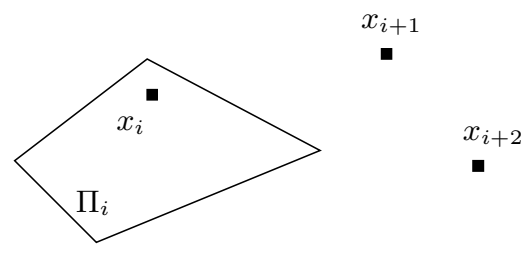

Fig. 1. Sensing Region of the $i^{\text {th }}$ sensor in $\mathbb{R}^{2}$

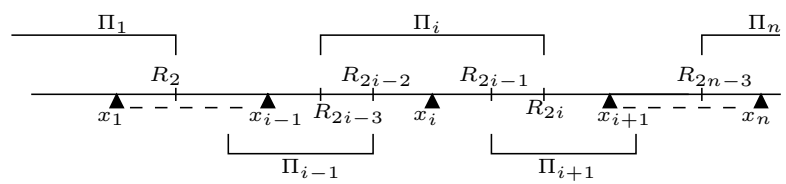

Fig. 2. Cooperative Sensing in $\mathbb{R}$

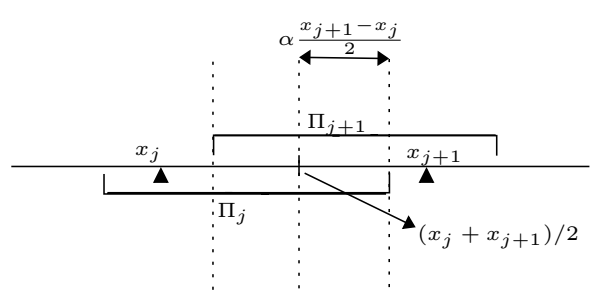

Fig. 3. Overlapping sensing regions

noise that resulted from a direct transmission from the $j^{\text {th }}$ node to the $i^{\text {th }}$ one. In this paper we take

$$
\sigma_{v_{i, j} \text { direct }}^{2}=\rho\left\|x_{i}-x_{j}\right\|^{2} \text {. }
$$

In general $\sigma_{v_{i, j, d i r e c t}}^{2}$ may also depend on the individual positions of the nodes. However, the slow variation of the received signal power, after averaging over fading and shadowing, is a non-increasing function of the distance between the nodes [18]. Therefore, to facilitate mathematical derivations in this paper, we take the communication noise variance as a distance-dependent function, as represented by Eq. 3. Alternatively, the communication noise variance could be thought of as the cost of communication (in terms of the required transmission power) in order to achieve a certain reception quality. Let $\alpha$ represent how communication quality compares with the quality of sensing. We take $\alpha=\frac{\rho}{\xi}$.

\section{B. Case of Perfect Communication: $\rho=0$}

If the communication among the nodes is perfect, then optimum sensor configuration can be characterized using Voronoi cells and can be achieved utilizing Lloyd algorithm. Here we briefly summarize the results. Readers are referred to [4] for the original results in the context of quantization. 
We will have the following for $\rho=0$ :

$$
\bar{D}_{i}=\sum_{j=1}^{n} \int_{x \in \Pi_{j}} f\left(\left\|x-x_{j}\right\|\right) p(x) d x, \quad 1 \leq i \leq n .
$$

Note that $\Pi_{i, j}=\Pi_{j}$ for $1 \leq i, j \leq n$ in this case. Given fixed sensor locations, optimum $\Pi_{i}$ will be as follows:

$$
\Pi_{i, \mathrm{opt}}=\left\{s \in Z \quad \mid \quad\left\|s-x_{i}\right\| \leq\left\|s-x_{j}\right\|, \forall j \neq i\right\},
$$

which means that the points that are closer to the $i^{\text {th }}$ sensor belong to $\Pi_{i}$. It can be seen that, for the ideal communication case, the region of interest is divided to non-overlapping sensing regions. Given fixed sensing regions, i.e. fixed $\Pi_{i} \mathrm{~s}$, each sensor should position itself at the center of mass of its region: $x_{i, \mathrm{opt}}=\frac{\int_{s \in \Pi_{i}} s \times p(s) d s}{\int_{s \in \Pi_{i}} p(s) d s}$. Lloyd algorithm provides an iterative attempt in reaching the optimum configuration [4]. At each time step, each sensor only needs to know the positions of its neighbors. It then finds its optimum sensing region using Eq. 5 and positions itself in the center of mass of the region. Following this procedure will guarantee convergence to the globally optimum configuration for logconcave $p($.$) and convex f($.$) functions [5].$

\section{Impact of a Non-Zero Communication Cost: $\rho \neq 0$}

Taking the distance-dependent communication cost into account can make the analysis different and more challenging for the following two reasons:

1) There will be overlapping sensing regions, i.e. $\Pi_{i} \bigcap \Pi_{j} \neq \emptyset$ for $i \neq j$. This means that there will be some regions in which two sensors rely on their own individual measurements, as opposed to one sensing and communicating its measurement to the other. To see this more clearly, consider the following example. Two students are studying for an exam together. Neither of the students has enough time to study the whole textbook individually. Therefore, they want to decide which chapters each student should learn on her won and which chapters she should learn from the other student. Communication cost, in this case, can be interpreted as the difficulty or the time it takes to transfer information from one student to the other. If the communication cost was zero, then the best strategy would have been to divide the chapters between the students based on their strengths. In such a case, each student would transfer her part to the other at no cost. There will then be no overlap in the material that students are studying individually. However, if there is a non-negligible communication cost associated with the transfer of information, there may be parts of the material that both students decide to study individually, as opposed to relying on learning them from the other student. This is the concept of overlapping sensing regions, which we will explore in a more mathematical framework in this paper.

2) Consider the case where the $j^{\text {th }}$ sensor needs to receive the measurement of the $i^{\text {th }}$ one. In general depending on the positions of the two sensors, direct transmission of the measurement may not be cost effective. Routing the information through other nodes makes more sense especially as other nodes may need to receive the same measurement. Therefore, the minimization of the overall estimation error variance, when $\rho \neq 0$, also necessitates optimization of the routing paths between any two nodes, which makes the analysis more challenging.

\section{Optimum SEnsing CONFIGURATION FOR $N=1$}

In this part, we analyze the problem of finding the optimum sensing regions and sensor positions for $N=$ 1. While a one-dimensional case may not represent real scenarios, it will establish the necessary foundation and give insights for more general cases. Fig. 2 shows a case where $n$ sensors are given the task of cooperatively estimating an event that will occur in $\mathbb{R}$. Without loss of generality, we label $x_{i}$ s from left to right, i.e. $x_{1}<x_{2}<\ldots<x_{n}$. $p(x)$ represents the probability density function of the event, where $x \in \mathbb{R}$. We will have

$$
\Pi_{i}=\left\{x \mid R_{2 i-3} \leq x \leq R_{2 i}\right\} \quad 1 \leq i \leq n,
$$

where $R_{i}$ s represent the boundaries of the sensing regions with $R_{-1}=R_{0}=-\infty \quad$ and $\quad R_{2 n-1}=R_{2 n}=\infty$.

Remark III.1: $\forall i R_{2 i} \geq R_{2 i-1}$.

Remark III.2: Depending on the communication and observation costs, there may exist an $i$ such that $R_{2 i-1}<$ $R_{2 i-2}$. Therefore, Fig. 2 shows one possible ordering of the $R_{i} \mathrm{~s}$.

Remark III.3: Consider the scenario in which the $(i+$ $m)^{\text {th }}$ sensor needs to send its observation to the $i^{\text {th }}$ sensor, where $m \geq 1$. It is easy to verify that $\left(x_{i+m}-x_{i}\right)^{2} \geq$ $\sum_{j=i+1}^{i+m}\left(x_{j}-x_{j-1}\right)^{2}$. Therefore the $(i+m)^{\text {th }}$ sensor should relay its measurement to the $i^{\text {th }}$ node through the nodes in between in order to minimize the overall estimation error variance. There is even more incentive for such relaying as the nodes in between, themselves, need to receive the observation of the $(i+m)^{\text {th }}$ sensor. Therefore, when an event occurs, every node needs to only communicate with its neighbors. We assume that the relaying nodes are merely passing on the information without any local processing.

Remark III.4: Consider an event that occurs in the overlapping region that is observed by both the $i^{\text {th }}$ and $(i+1)^{\text {th }}$ sensors: $x \in \Pi_{i} \bigcap \Pi_{i+1}$. Then the $j^{\text {th }}$ sensor should rely on receiving the measurement of that event from whichever sensor that is closer to it in order to minimize $\bar{D}$. This can be easily verified using the definition of sensing regions and the relaying strategy discussed in Remark III.3.

\section{A. Estimation Error Variance}

Let $D_{i}(x)$ represent the estimation error variance of the $i^{\text {th }}$ sensor given that an event has occurred at position $x . \bar{D}_{i}$ will then represent the average of $D_{i}(x)$ over the distribution of the event. The average estimation error variance can then be written as follows:

$$
\bar{D}_{i}=\bar{D}_{i, \text { center }}+\bar{D}_{i, \text { up }}+\bar{D}_{i, \text { down }}, \text { where, }
$$


$\bar{D}_{i, \text { center }}=\int_{R_{2 i-3}}^{R_{2 i}} D_{i}(x) p(x) d x=\xi \int_{R_{2 i-3}}^{R_{2 i}}\left(x-x_{i}\right)^{2} p(x) d x$.

Since each sensor receives the measurements of other nodes through communicating with its neighbors, we will have:

$$
\begin{aligned}
\bar{D}_{i, \text { up }} & =\int_{R_{2 i}}^{\infty} D_{i}(x) p(x) d x \\
& =\xi \int_{R_{2 i}}^{R_{2 i+2}}\left(x-x_{i+1}\right)^{2} p(x) d x \\
& +\bar{D}_{i+1, \text { up }}+\rho\left(x_{i+1}-x_{i}\right)^{2} \int_{R_{2 i}}^{\infty} p(x) d x \text { and } \\
\bar{D}_{i, \text { down }} & =\int_{-\infty}^{R_{2 i-3}} D_{i}(x) p(x) d x \\
& =\xi \int_{R_{2 i-5}}^{R_{2 i-3}}\left(x-x_{i-1}\right)^{2} p(x) d x \\
& +\bar{D}_{i-1, \text { down }}+\rho\left(x_{i}-x_{i-1}\right)^{2} \int_{-\infty}^{R_{2 i-3}} p(x) d x .
\end{aligned}
$$

We will have the following for the overall estimation error variance, noting that $\bar{D}_{n, \text { up }}=\bar{D}_{1, \text { down }}=0$,

$$
\begin{aligned}
\bar{D} & =\xi \sum_{i=1}^{n} \int_{R_{2 i-3}}^{R_{2 i}}\left(x-x_{i}\right)^{2} p(x) d x \\
& +\xi \sum_{i=1}^{n-1} \sum_{k=i}^{n-1} \int_{R_{2 k}}^{R_{2 k+2}}\left(x-x_{k+1}\right)^{2} p(x) d x \\
& +\rho \sum_{i=1}^{n-1} \sum_{k=i}^{n-1}\left(x_{k+1}-x_{k}\right)^{2} \int_{R_{2 k}}^{\infty} p(x) d x \\
& +\xi \sum_{i=2}^{n} \sum_{k=2}^{i} \int_{R_{2 k-5}}^{R_{2 k-3}}\left(x-x_{k-1}\right)^{2} p(x) d x \\
& +\rho \sum_{i=2}^{n} \sum_{k=2}^{i}\left(x_{k}-x_{k-1}\right)^{2} \int_{-\infty}^{R_{2 k-3}} p(x) d x .
\end{aligned}
$$

\section{B. Optimum Coverage Regions}

Given fixed sensor locations, we will have the following for the optimum $R_{2 j}$ and $R_{2 j-1}:^{2}$

$$
\begin{gathered}
\frac{\partial \bar{D}}{\partial R_{2 j}}=0 \text { for } 1 \leq j \leq n-1 \Longrightarrow \\
\left(R_{2 j}-x_{j}\right)^{2}+(j-1)\left(R_{2 j}-x_{j}\right)^{2}= \\
\alpha j\left(x_{j+1}-x_{j}\right)^{2}+j\left(R_{2 j}-x_{j+1}\right)^{2},
\end{gathered}
$$

which results in

$$
R_{2 j, \mathrm{opt}}=\frac{x_{j}+x_{j+1}}{2}+\alpha \frac{x_{j+1}-x_{j}}{2} \quad 1 \leq j \leq n-1 .
$$

Similarly, $\frac{\partial \bar{D}}{\partial R_{2 j-1}}=0$ for $1 \leq j \leq n-1 \Longrightarrow$

$$
R_{2 j-1, \mathrm{opt}}=\frac{x_{j}+x_{j+1}}{2}-\alpha \frac{x_{j+1}-x_{j}}{2} \quad 1 \leq j \leq n-1 .
$$

\footnotetext{
${ }^{2}$ Note that in this paper, we are not analyzing global optimality of the derived configuration. Such analyses are important and are among possible future extensions of this work.
}

Note that $\frac{x_{j}+x_{j+1}}{2}$ represent the optimum boundaries for the ideal communication case. We can then see that, in the non-ideal communication case, the optimum boundaries are shifted to the left and right by $\alpha \frac{x_{j+1}-x_{j}}{2}$ to form the overlapping sensing regions. This is shown in Fig. 3.

\section{Optimum Sensor Positions}

Similarly, it can be easily shown that given $R_{j} \mathrm{~s}$, the optimum sensor positions are the solution to the following set of linear equations for $1 \leq j \leq n$ :

$$
\frac{\partial D}{\partial x_{j}}=0 \Longrightarrow \lambda_{j} x_{j, \mathrm{opt}}+\beta_{j} x_{j-1, \mathrm{opt}}+\gamma_{j} x_{j+1, \mathrm{opt}}=\eta_{j},
$$

with $x_{0}=x_{n+1}=0$,

$$
\begin{aligned}
\lambda_{j} & =\int_{R_{2 j-3}}^{R_{2 j}} p(x) d x+(j-1) \int_{R_{2 j-2}}^{R_{2 j}} p(x) d x \\
& +(n-j) \int_{R_{2 j-3}}^{R_{2 j-1}} p(x) d x+\alpha(j-1) \int_{R_{2 j-2}}^{\infty} p(x) d x \\
& +\alpha j \int_{R_{2 j}}^{\infty} p(x) d x+\alpha(n-j+1) \int_{-\infty}^{R_{2 j-3}} p(x) d x \\
& +\alpha(n-j) \int_{-\infty}^{R_{2 j-1}} p(x) d x, \\
\beta_{j} & =-\alpha(j-1) \int_{R_{2 j-2}}^{\infty} p(x) d x \\
& -\alpha(n-j+1) \int_{-\infty}^{R_{2 j-3}} p(x) d x, \\
\eta_{j} & =\int_{R_{2 j-3}}^{R_{2 j}} x p(x) d x+(j-1) \int_{R_{2 j-2}}^{R_{2 j}} x p(x) d x \\
& +(n-j) \int_{R_{2 j-3}}^{R_{2 j-1}} x p(x) d x \text { and } \gamma_{j}=\beta_{j+1} .
\end{aligned}
$$

\section{Achieving the Optimum Configuration in a Distributed Manner}

In the previous section we derived optimum sensor positions and coverage regions in the presence of non-ideal communication links. In this part, we are interested in achieving the optimum solution, starting from any initial positions, in a distributed manner. By "distributed" we are referring to the case in which each sensor only communicates to its two neighbors. Then it makes an independent decision on its coverage area and position. At fixed sensor positions, the optimum coverage areas are already in a distributed form, as can be seen from Eq. 12 and 13. Each sensor only needs to know the positions of its two neighbors to find its optimum coverage area. Given the coverage areas, each sensor then has to position itself at the optimum location, which is the solution to the linear set of equations denoted by Eq. 14. It is possible to solve these equations to find $x_{i, \mathrm{opt}}$. However, the direct solution will require the $i^{\text {th }}$ node to know more than the locations of its two neighbors. Due to the structure of this set of linear equations, however, it is possible to achieve the optimum solution in a distributed manner iteratively. Let 
$x_{i}^{k}$ represent the position of the $i^{\text {th }}$ sensor at $k^{\text {th }}$ iteration. Then the $i^{\text {th }}$ sensor will communicate with its neighbors to receive their positions, $x_{i-1}^{(k)}$ and $x_{i+1}^{(k)}$. Based on the received information, it will go to the following position: ${ }^{3}$

$$
\begin{gathered}
x_{i}^{(k+1)}=-\frac{\beta_{i}}{\lambda_{i}} x_{i-1}^{(k)}-\frac{\gamma_{i}}{\lambda_{i}} x_{i+1}^{(k)}+\frac{\eta_{i}}{\lambda_{i}} \Longrightarrow \\
X^{(k+1)}=A X^{(k)}+B,
\end{gathered}
$$

where $X=\left[\begin{array}{llll}x_{1} & x_{2} & \ldots & x_{n}\end{array}\right]^{T}$, all the elements of $A$ are zero expect for $A(i, i+1)=-\frac{\gamma_{i}}{\lambda_{i}}$ and $A(i+1, i)=$ $-\frac{\beta_{i+1}}{\lambda_{i+1}}$ for $1 \leq i \leq n-1$. Furthermore, $B(i)=\frac{\eta_{i}}{\lambda_{i}}$ for $1 \leq$ $i \leq n$ and $X^{(k)}$ represents $X$ at $k^{\text {th }}$ iteration. It can be easily confirmed that Eq. 14 has a unique solution as $I_{n}-A$ is strictly diagonally dominant, where $I_{n}$ is an identity matrix.

Remark III.5: It can be easliy confirmed that the $i^{\text {th }}$ sensor can calculate $\beta_{i}, \lambda_{i}, \gamma_{i}$ and $\eta_{i}$ locally, based on its own position and the communicated positions of its two neighbors.

Lemma 1 (Gershgorin Disk Theorem) [19]: Let $C$ represent an $n \times n$ matrix with entries $C(i, j)$. Then each of the non-zero eigenvalues of $C$ is in at least one of the following disks: $\left\{s \in \mathbb{R}^{2}:|s-C(i, i)| \leq \sum_{j, j \neq i} C(i, j)\right\}$.

Let $X_{\mathrm{opt}}=\left[\begin{array}{llll}x_{1, \mathrm{opt}} & x_{2, \mathrm{opt}} & \ldots & x_{n, \mathrm{opt}}\end{array}\right]^{T}$ and $\epsilon^{(k)}=$ $X^{(k)}-X_{\text {opt }}$. We will have $\epsilon^{(k)}=A^{k} \epsilon^{(0)}$. It can be easily confirmed, using the definitions of $\beta_{i}, \lambda_{i}, \gamma_{i}$ and $\eta_{i}$, that $\max \left\{\frac{\left|\gamma_{i}\right|+\left|\beta_{i}\right|}{\left|\lambda_{i}\right|}\right\}<1$. Let $r$ represent spectral radius of matrix $A$. Using Lemma 1, we will have $r \leq \max \left\{\frac{\left|\gamma_{i}\right|+\left|\beta_{i}\right|}{\left|\lambda_{i}\right|}\right\}$. Therefore $r<1$, resulting in $\lim _{k \rightarrow \infty} X^{(k)} \rightarrow X_{\text {opt }}$.

Starting from any initial positions, the sensors can achieve the optimum configuration in a distributed manner by following these steps:

At each iteration,

1) Each node communicates its position to its neighbors

2) using the communicated information, each node identifies its optimum coverage area based on Eq. 12 and 13

3) The nodes will move to the optimum positions, indicated by the solution of Eq. 14, by following these subiterations:

3a) Each node communicates its position to its neighbors

3b) Each node updates its position based on Eq. 16

3c) Proceed to $3 \mathrm{a}$ or terminate

4) Proceed to 1 or terminate.

\section{E. Simulation Results}

To see the performance of the proposed distributed algorithm, Fig. 4 shows convergence of the positions of 7 sensors to the optimum configuration for $\alpha=2$. The probability density function of the event of interest is taken to be a zero-mean Gaussian distribution with the variance

\footnotetext{
${ }^{3}$ Note that $x_{i}$ will receive noisy versions of $x_{i-1}$ and $x_{i+1}$, due to communication noise. We will consider the effect of such noisy samples in the next section.
}

of $100(\sigma=10)$ for this example. The nodes are initiated at random locations. It can be seen that the sensors converge to the optimum configuration after a few iterations. To see the impact of the quality of the communication links on the performance, Fig. 5 shows $\bar{D}$, the overall estimation error variance, for $\xi=1, \alpha=2$ and 0.2 , and for different number of sensors. It can be seen that quality of the communication links can affect the performance considerably.

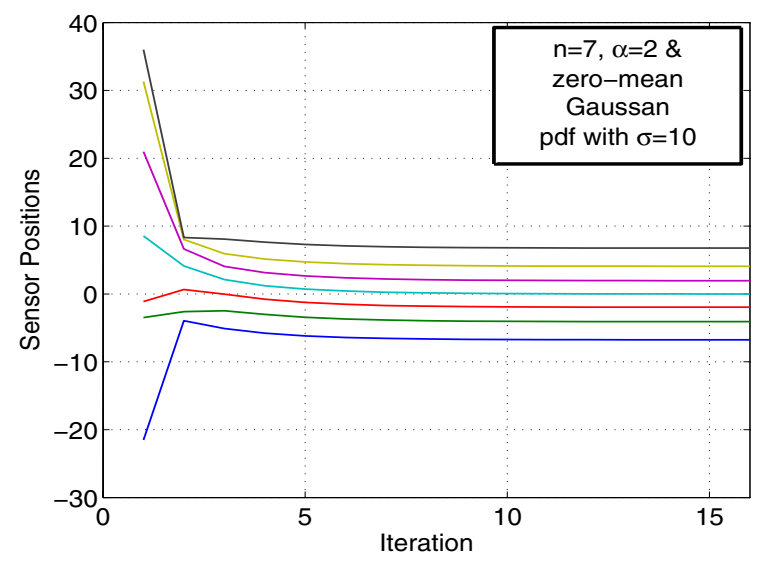

Fig. 4. Performance of the proposed distributed algorithm - convergence of 7 sensors from random initial positions to the optimum configuration in a distributed manner

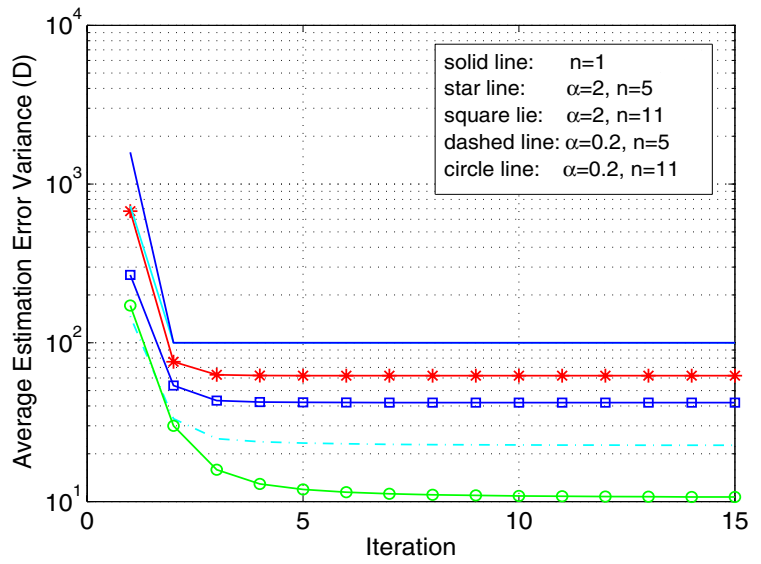

Fig. 5. Impact of comm. links on overall estimation error variance, $\xi=1$

\section{F. Impact of Communication Noise on the Transmitted Positions}

In Section III-A, B and C, we have addressed the impact of communication noise on distributed sensing and estimation by finding the optimum sensing regions and sensor positions in the presence of non-ideal communication links. In Section III-D, we proposed a distributed way of achieving the optimum configuration, where each node only needed to communicate to its neighbors to receive their positions. We did not, however, consider the impact of communication 
noise on the communicated positions in Section III-D. Let $\hat{x}_{i, i-1}$ and $\hat{x}_{i, i+1}$ represent the reception of the $i^{\text {th }}$ sensor when the $i-1$ and $i+1$ sensors transmit their positions respectively. Then Eq. 16 will change as follows:

$$
x_{i}^{(k+1)}=-\frac{\beta_{i}}{\lambda_{i}} \hat{x}_{i, i-1}^{(k)}-\frac{\gamma_{i}}{\lambda_{i}} \hat{x}_{i, i+1}^{(k)}+\frac{\eta_{i}}{\lambda_{i}} .
$$

Similarly, the $i^{\text {th }}$ node uses $\hat{x}_{i, i-1}$ and $\hat{x}_{i, i+1}$ to determine its optimum coverage region in every step.

To limit the amount of noise in the received samples, each sensor monitors the quality of its communication links to its neighbors (through measuring the received Signal to Noise Ratio for instance). If the quality of a link is below a certain threshold, the receiver ignores the communication. For instance consider two nodes that are initially located considerably far from each other and want to find the optimum configuration. If the communication noise is excessively high, each sensor ignores the transmission of the other. This means that each sensor will act individually by moving towards the center of mass of the distribution (optimum solution for $n=1$ ). The quality of the communication link improves as they move towards the center of mass. At some point, the link quality becomes acceptable and the nodes proceed to use the transmitted information. Fig. 6 shows the convergence of the proposed distributed algorithm considering the communication noise in the received positions. As can be seen, even for a considerably high noise variance, $\rho=0.1$, the algorithm performs considerably well.

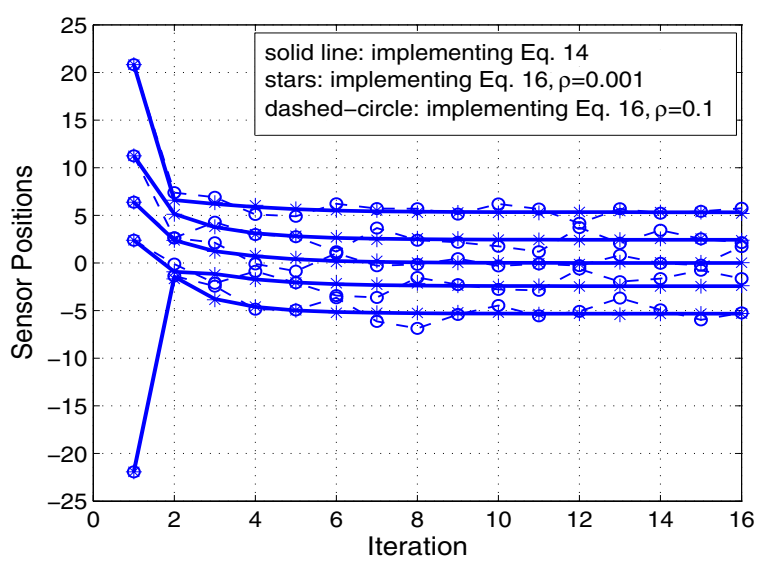

Fig. 6. Effect of noisy samples on convergence of the proposed algorithm, $n=5, \alpha=2$, zero-mean Gaussian pdf with $\sigma=10$

\section{Summary AND Future Work}

In this paper we considered distributed sensing and estimation under communication constraints in mobile networks. We derived expressions for optimum sensing regions and sensor positions when considering imperfect communication links. The results indicated that the optimum configuration consists of overlapping sensing regions. We also proposed a distributed algorithm to achieve the optimum configuration from any initial positions. The analysis was provided for a one-dimensional case. We are currently working on extending the analysis to $\mathbb{R}^{2}$. We also did not provide the conditions under which the configuration, found in Section III, would be globally optimal. Characterizing such conditions is another possible future direction. We also assumed communication and observation noise variances that are proportional to the distance square. It is important to consider other non-decreasing functions. We are also working on extending the results to multiple targets. Finally, we did not consider target mobility. For mobile targets, it becomes important to find optimum trajectories and distributed ways of achieving them considering non-ideal communication links.

\section{REFERENCES}

[1] Z. Drezner, Ed., "Facility Location: A Survey of Applications and Methods, Springer Series in Operations Research. Springer Verlag, NewYork, NY, 1995.

[2] A. Okabe and A. Suzuki, "Locational optimization problems solved through Voronoi diagrams", European Journal of Operational Research, vol. 98, no. 3, pp.445-56, 1997

[3] Q. Du, V. Faber, M. Gunzburger, "Centroidal Voronoi Tessellations: Applications and Algorithms," SIAM Review, vol. 41, no. 4, 1999

[4] S. Lloyd, "Least squares quantization in PCM," IEEE Transactions on Information Theory, Vol. 28, Issue 2, March 1982 Page(s):129 - 137

[5] J. Kieffer, "Uniqueness of locally optimal quantizer for log-concave density and convex error weighting function," IEEE Transactions on Information Theory, volume 29, Issue 1, Jan 1983 Page(s):42 - 47

[6] J. Cortes, S. Martinez, T. Karatas and F. Bullo, "Coverage control for mobile sensing networks," IEEE Transactions on Robotics and Automation, Volume 20, Issue 2, April 2004, Page(s):243 - 255

[7] B.Sinopoli, L. Schenato, M. Franceschetti, K. Poolla, M. Jordan, S., "Kalman filtering with intermittent observations," IEEE Transactions on Automatic Control, Vol. 49, Issue: 9, Sept. 2004

[8] X. Liu and A. J. Goldsmith, "Kalman Filtering with Partial Observation Losses," 43rd IEEE Conference on Decision and Control, 2004

[9] Y. Mostofi and R. Murray, "On Dropping Noisy Packets in Kalman Filtering Over a Wireless Fading Channel," 24th American Control Conference (ACC), 2006

[10] Y. Mostofi and R. Murray, "New Design Principles for Estimation over Fading Channels in Mobile Sensor Networks," 44th IEEE Conference on Decision and Control (CDC), 2005

[11] Y. Mostofi and R. Murray, "Effect of Time-Varying Fading Channels on the Control Performance of a Mobile Sensor Node," Proceedings of 1st IEEE International Conference on Sensor and Adhoc Communications and Networks, 2004

[12] L. Xiao, M. Johansson, H. Hindi, S. Boyd and A. Goldsmith, "Joint optimization of communication rates and linear systems," IEEE trans. on automatic control, vol. 48, no. 1, Jan. 2003

[13] X. Liu and A. Goldsmith, "Wireless communication tradeoffs in distributed control," 42nd IEEE Conference on Decision and Control, volume: 1, 2003, pages:688 - 694

[14] X. Liu and A. J. Goldsmith, "Wireless Medium Access Control in Distributed Control Systems," Allerton Conference on Communication, Control and Computing, 2003

[15] S. Tatikonda, A. Sahai and S. Mitter, "Control of LQG systems under communication constraints," IEEE 37th Conference on Decision and Control, 1998, vol. 1, Pages:1165-1170

[16] J. Cortes, S. Martinez and F. Bullo, "Spatially-distributed coverage optimization and control with limited-range interactions," ESAIM: Control, Optimisation and Calculus of Variations 11 (4) (2005)

[17] Y. Mostofi, T. Chung, R. Murray and J. Burdick, "Communication and Sensing Trade Offs in Decentralized Mobile Sensor Networks: A Cross-Layer Design Approach," 4th International Conference on Information Processing in Sensor Networks (IPSN), 2005

[18] W. Jakes, Microwave Mobile Communications. IEEE Press, 1974

[19] R. Horn and C. Johnson, Matrix Analysis. Cambridge University Press 1999 